NICE guidelines

\section{The NICE guidelines for the management of head injury: the view from a district hospital}

\section{A M Leaman}

\section{Compelling scientific evidence is required before implementation of new forms of health care}

I n June 2003 the National Institute for Clinical Excellence (NICE) issued guidance on the management of head injuries in the UK. The key features of this guidance were that the indications for computed tomography (CT) should be widened and that CT should replace skull radiography in the investigation of minor head injury. Reaction among my colleagues to these guidelines ranged from despair to disbelief.

Despair because this is yet another piece of central guidance that has been issued with little regard for its operational or financial consequences.

For example, an initial assessment of the impact of this guidance in this hospital indicates that an additional 725 CT scans will be required each year, of which about half will be performed outside office hours. Given that there is a national shortage of radiologists, is it a good idea to promote a policy that demands so much additional radiologist input?

Data issued by the National Radiological Protection Board also indicate that a CT head scan exposes a person to 100 chest radiographs' worth of radiation, ${ }^{1}$ and the NRPB has an ongoing campaign to reduce the public's exposure to medical radiation. How does this square with a policy that would dramatically increase the use of head CT, particularly affecting the brains of young children?

The implementation of these guidelines will also result in patients waiting long periods in A\&E for a scan. How will this help A\&E departments seeking to achieve the four hour throughput target set by the government? The guidance also encourages patients with minor head injury to dial 999 for an ambulance. What impact will this have on our already hard pressed ambulance ser- vices? So much for "joined up" thinking by the NICE advisory panel.

Predictably the NICE guidance does not come with any additional funding, and in this hospital alone the estimated cost of implementation is $£ 30000$. Multiply that by 500 to get some idea of the total cost to the health service.

There is also disbelief. Firstly, because the problem. On a Sunday afternoon most A\&E departments are full of children who have fallen a metre or so and banged their head, or who have vomited after a head injury. How many of these children are going to have a CT scan, and how many will need a general anaesthetic to allow this to be performed?

Furthermore, in minor head injury one of the most common abnormalities found on CT is skull fracture. ${ }^{2}$ Given that such fractures are usually visible on skull radiography is there really no future for this inexpensive and low radiation investigation?

The guidance also suggests that normal CT will save beds by avoiding admission. What is the evidence for this, and will A\&E departments really send home badly concussed patients just because their CT scan is normal?

All these doubts call into question the scientific basis, and the authorship, of the NICE guidance. Unfortunately the written guidelines issued by NICE do not quote the articles upon which this advice is based. Surely we have moved on from the days when "experts" hand down instructions without providing the supportive evidence. Furthermore, many of the key recommendations are based on level 5 "evidence" - that is, expert opinion only. As to the authorship of the guidance, it would appear that very few of the NICE experts the guidance seems disproportionate to manage minor head injuries on a day to day basis. Perhaps this explains the underlying impracticability of much of their advice.

It is therefore apparent that an objective scientific analysis is required of the NICE guidelines for head injury. Much of the evidence upon which this guidance is based comes from tertiary trauma centres in North America. The case mix at these units will be skewed towards more serious injury, and is likely to be quite different to the sort of head injuries seen in most UK A\&E departments. There is a parallel here with trauma centres. In the 1980s, publications from the US seemed to suggest that trauma centres should be established in the UK. But when a formal trial was carried out here no appreciable benefit was demonstrated. ${ }^{3}$

If the scientific evidence concerning CT minor head injuries can be shown to be applicable to this side of the Atlantic, then a proper cost/benefit analysis should be carried out to assess the probable impact of this guidance in UK hospitals. This analysis should include the radiation risks ${ }^{4}$ and the practical difficulties of $\mathrm{CT}$, particularly in children. Such an analysis could save the health service a lot of money and prevent excessive exposure to radiation.

Ultimately this issue calls into question whether it is either helpful or even possible for a group of "experts" to dictate medical practice. Patient's interests are best served if their doctor questions received wisdom, and demand compelling scientific evidence before implementing new forms of health care.

Emerg Med J 2004;21:400.

doi: $10.1136 / \mathrm{emj} .2004 .015024$

Correspondence to: Mr A Leaman, Accident and Emergency Department, Princess Royal Hospital, Telford TF1 6TF, UK; caleaman@ doctors.org.uk

Funding: none.

Competing interests: none declared.

\section{REFERENCES}

1 National Radiological Protection Board. http:// www.nrpb.org/radiation_topics/medical/ ted_equivalent. $h$ tm

2 Nagy KK, Joseph KT, Krosner SM, et al. The utility of head computed tomography after minimal head injury. J Trauma 1999;46:268-70.

3 Nicholl J, Turner J. The effectiveness of a regional trauma system in reducing mortality from major trauma: before and after study. BMJ 1997;315:1349-54.

4 Khursheed A, Hillier MC, Shrimpton PC, et al. Influence of patient age on normalised effective doses calculated for CT examinations. Br J Radiol 2002;75:818-30. 
NICE guidelines

\section{Are we ready for NICE head injury guidelines in Scotland?}

\section{J Swann, T Kelliher, J Kerr}

\section{The major challenge for A\&E is implementation of realistic guidelines}

$\Lambda$ ny guideline that gives priority to the prompt identification of patients at risk of brain injury deserves to be supported and timely imaging (CT brain scan or skull radiograph, or both) is an essential part of this process. Recently published guidelines recognise the increased availability of emergency CT in the UK. ${ }^{1-3}$ The Scottish Intercollegiate Guidelines Network Publication number 46 (August 2000) recommends the increased use of CT brain scans for selected patients with mild head injury including those with the radiological demonstration of a skull fracture. ${ }^{2}$

The National Institute for Clinical Excellence: Clinical Guideline $(2003)^{3}$ includes an adapted form of The Canadian CT head rules, ${ }^{4}$ which lowers the threshold for scanning patients with mild head injury much further and relies very little on the use of skull radiographs.

A concern is whether such guidelines can be safely and effectively implemented if hospitals in the UK lack the necessary resources for easy access to out of hours CT.

The Canadian CT head rules were based on the study of patients who had a history of loss of consciousness or post-traumatic amnesia after blunt head injury. In Steill's paper, the population studied from the 10 Canadian hospitals appears to be less violent than that of some major inner city A\&E departments in the UK. Most of the injuries were attributable to falls or road traffic accidents. Of the $11 \%$ attributable to assault most were by use of hands or feet rather than blunt objects and only $8 \%$ of assaults suffered significant "brain injury" as evident on CT scans.

In the UK attempts to establish the probable impact of the NICE guideline are underway and not surprisingly indicate that there would be a significant increase in the CT scan rate if the guideline was adhered to. It remains to be seen how well individual hospitals can cope with the increased demand for CT scans.
NICE recommend immediate request for CT of patients who still have depressed GCS (14/15) two hours after injury. This should have support from those responsible for the observation of such patients on wards if it detects those patients needing referral to a neurosurgical unit while they are still in the A\&E department. Clinical variables such as coagulopathy and vomiting (twice) are rightly included as warranting a low threshold for CT but the emergency scanning of all patients over 64 years of age with any amnesia may prove difficult to implement.

Most head injured patients are GCS 15 on arrival in A\&E, with little or no post-traumatic amnesia or primary brain damage. If they have a skull fracture their risk of needing an operation for intracranial haematoma is increased several hundred times. ${ }^{5}{ }^{6}$ In most Scottish A\&E departments skull radiographs are still used as a screening method to detect skull fractures in mild head injury (GCS13-15). Nevertheless, in the past few years since the development of the SIGN guideline there has been a significant increase in the utilisation of CT brain scans, some instead of and some after skull radiography.

In accordance with the SIGN guideline, we believe there is still a role for skull radiological examination, for example, in patients with a significant mechanism of injury who may have sustained a depressed skull fracture attributable to blunt or penetrating trauma. We are concerned that, if NICE guidelines are followed, A\&E doctors may not request CT scans until such patients deteriorate from intracranial haematoma or brain injury.

Ultimately, the most contentious aspect of the NICE guidelines is their advising an immediate CT scan in any patient who has been GCS 12 or less at any point since injury. This would include many patients who in retrospect have simply been intoxicated or have other reversible causes of depressed consciousness and may result in a large proportion of unnecessary scans.
Furthermore, from a logistical viewpoint, if it were agreed that all such patients did require a CT scan, most radiology services would be unable to cope with the workload. It is ironic that The London Royal College of Radiologists recommends the Canadian CT head rules and yet their members are often not in a position to comply with their implementation! ${ }^{7}$

Some lowering of the threshold for early CT is welcome but not to the degree or in the form required by NICE. While the content of the SIGN guideline is not perfect it is increasingly accepted by radiologists and clinicians and we would recommend it as a more realistic alternative to NICE. If further improvement in the head injury service is to be achieved, the major challenge for A\&E is implementation of realistic guidelines. This requires education of medical and nursing staff with an emphasis on early selection for imaging, frequent charting of observations, and improved documentation.

\section{ACKNOWLEDGEMENTS}

This view is not just a personal one but reflects recent discussion with A\&E, radiology, and neurosurgery colleagues in Scotland. We are particularly grateful to our A\&E colleagues Patrick Grant (Western Infirmary Glasgow), John Hiscox (Aberdeen Royal Infirmary), Colin Robertson (Edinburgh Royal Infirmary), Michael Johnston (Ninewells Hospital, Dundee), to radiologists, Douglas McCarter Glasgow Royal Infirmary; Scott McKie and Paul Allan, Edinburgh Royal Infirmary; neurosurgeons Laurence Dunn and Graham Teasdale (INS, Southern General Hospital, Glasgow) for their views.

Emerg Med J 2004;21:401-402.

doi: 10.1136/emj.2004.015644

\section{Authors' affiliations}

I J Swann, T Kelliher, Department of Accident and Emergency Medicine, Glasgow Royal Infirmary, Glasgow, UK

J Kerr, Accident and Emergency Department, Royal Infirmary of Edinburgh, Edinburgh, UK

Correspondence to: Mr I J Swann, Department of Accident and Emergency Medicine, Glasgow Royal Infirmary, Glasgow G4 OSF, UK; swann@campsierd.fsnet.co.uk

\section{REFERENCES}

1 Royal College of Surgeons of England. Report of the working party on the management of patients with head injuries. London: RCS, 1999.

2 Scottish Intercollegiate Guidelines Network. Early management of patients with a head injury. Edinburgh: SIGN Secretariat, Royal College of Physicians, 2000

3 National Institute for Clinical Excellence Head injury triage, assessment, investigation and early management of head injury in infants, children and adults. Clinical guideline 4 June 2003 (http://www.nice.org/).

4 Steill IG, Wells GA, Vandemheen K, et al. The Canadian CT head rule for patients with minor head injury. Lancet 2001;357:1391-6. 
5 Teasdale GM, Murray G, Anderson E, et al. Risks of acute traumatic intracranial haematoma in children and adults: implications for management of head injuries. BMJ 1990;300:363-7.
6 Mendelow AD, Teasdale G, Jennett B, et al. Risks of intracranial haematoma in head injured adults. BMJ 1983;287:1173-6.
7 The Royal College of Radiologists. Making the best use of a department of clinical radiology. Guidelines for doctors. 5th edn. London: RCR, 2003:96-9.
NICE guidelines

\section{NICE head injury guidelines}

\section{T J Coats}

\section{Emergency physicians in the UK will be scanning more patients with head injury and should have easier access to $C T$}

$\mathrm{T}$ e current discussion about the NICE head injury guidelines illustrates both the strengths and the weaknesses of the system of national guidelines for the NHS. We are still on the learning curve of how we should use this national advice in emergency medicine practice. When there is good evidence that tells that a treatment works or that a treatment does not work a guideline is easy to write. However, when there is weak evidence, as the research has not been done, writing a guideline becomes more difficult. ${ }^{1}$ Simply leaving a gap in the guideline would not be useful, however the "best evidence available" becomes a consensus opinion among experts. Different groups of experts may come to different opinions and an individual emergency physician may, because of personal clinical experiences or particular local circumstances, disagree with the consensus. Guideline developers recognise that there is no way of telling who is right and who is wrong (until further research is conducted) and so label consensus opinions with the lowest level of evidence (grade D).

Each step in a guideline should therefore be regarded rather differently. Much more weight should be given to the parts of a guideline supported by higher grade evidence, as we are more certain that this is the right thing to do for the average patient. The parts supported by grade D evidence are much more open to question and modification to fit an individual patient. We should not think that once a guideline has been developed it should be slavishly followed in all circumstances. Unfortunately when guidelines are discussed, flow diagrams constructed, or departmental guidelines written, the underpinning evidence becomes removed and all steps look as if they have the same weight.
Clinical experience also comes into play here. I would expect an emergency department SHO to closely follow a guideline. However, I would also expect an experienced emergency physician to know which parts of a guideline are based on weaker evidence and to exercise more clinical skill in these areas, so that management is tailored to the individual patient. Our audit systems also need to become more sophisticated in the way that they use guidelines to define "right" and "wrong" treatment, although I doubt that the politicians (and lawyers) will be weaned from their belief that medicine is full of certainties. Simply ticking the box that says "followed the NICE head injury guideline" does not take into account the subtleties of individual patient management and will not provide meaningful audit or a good assessment of performance.

It is debatable how the availability of resources should influence the development of a guideline. The inability of a hospital to provide a computed tomography (CT) service that can cope with the NICE head injury guidelines seems to be a strong argument for improving the service rather than changing the guideline. The burden on radiologists does not need to be great as there is no reason why emergency physicians should not interpret a CT head scan, which can be less difficult than interpreting a chest radiograph.

In the UK the greatest effect of the new guidelines may be an improved access to CT for all head injured patients. It is rather ironic that the Canadian CT head rules were used in North America to decrease the number of CT scans performed on minor head injuries, whereas in the UK they will have the opposite effect. Estimates of the numbers of additional CT scans in the "average $\mathrm{ED}^{\prime \prime}$ seem to vary from 48 a year $^{2}$ to 725 a year. ${ }^{3}$ Experience from the introduction of a guideline similar to NICE is particularly important, ${ }^{4}$ and indicates that the upper figure is wrong. The Cambridge experience also questions whether admissions will be reduced-it will interesting to see if the admission rate falls as more experience is accumulated and we become confident with the new approach.

In the absence of comprehensive evidence guidelines are always going to be imperfect. The group that developed the NICE guidance consulted widely among practising emergency clinicians and has been transparent about the details of the evidence on which the guidance is based (full details are on the NICE web site but not in the printed format). There are a number of areas for future research-some of which is already underway. This has been acknowledged in the short interval before the guidelines are due for a review (June of next year). There seems to be a consensus that some lowering of the threshold for CT is desirable, ${ }^{5}$ but uncertainty about management of some subgroups. The details can be debated, but the underlying messages of the NICE head injury guidelines-that emergency physicians in the UK will be scanning more patients with head injury and will have easier access to CT-should be endorsed.

Emerg Med J 2004;21:402. doi: 10.1136/emj.2004.016485

Correspondence to: Professor T J Coats, Leicester Royal Infirmary, Infirmary Square, Leicester LE1 5WW, UK; t.coats@virgin.net

Funding: none.

Conflicts of interest: none declared.

\section{REFERENCES}

1 Dunning J, Lecky F. The NICE guidelines in the real world: a practical perspective. Emerg Med J 2004;21:404-7.

2 Shravat BP, Hynes KA. The impact of NICE guidelines for the management of head injury on the workload of the radiology department. Emerg Med J 2004;21:521-2.

3 Leaman AM. The NICE guidelines for the management of head injury: the view from a district hospital. Emerg Med J 2004;21:400.

4 Sultan HY, Boyle A, Pereira M, et al. Application of the Canadian CT head rules in managing minor head injuries in a UK emergency department: implications for the implementation of the NICE guidelines. Emerg Med J 2004;21:420-5.

5 Swann IJ, Kelliher T, Kerr J. Are we ready for NICE head injury guidelines in Scotland? Emerg Med J 2004;21:401-2. 
HIV post-exposure prohpylaxis

\section{HIV post-exposure prophylaxis after sexual assault}

\section{J Crossley}

\section{The decision for HIV post-exposure prophylaxis after sexual assault should be considered on an individual basis}

M erchant and colleagues report an audit of adherence in their paediatric emergency department to the New York State Department of Health guidelines on HIV post-exposure prophylaxis (PEP). ${ }^{1}$ The guidelines recommend that every victim of unprotected vaginal or anal rape presenting within 36 hours should receive HIV PEP unless the assailant is known to be HIV negative. Where possible, they should receive treatment within one hour of assault. Over six months, 14 of 25 patients received HIV PEP. No patient presented within one hour. Even after presentation, the minimum triage to medication time was two hours. The authors suggest strategies to speed the delivery of prophylaxis.

Many of the important questions come well before an audit of adherence to guidelines. What are the risks associated with sexual assault? What are the risks and benefits of HIV PEP?

These are thorny issues, and it is notable that only a minority of the states in the USA have produced guidelines concerning HIV PEP in adolescents. In the UK there are no national guidelines. In 2002 the Medical Society for the Study of Venereal Diseases surveyed opinion and practice in genitourinary medicine clinics across the UK. ${ }^{2}$ Since 1997, requests and prescriptions for HIV PEP had risen fourfold to 242 and sevenfold to 130 respectively. Most clinicians surveyed would prescribe prophylaxis where a known HIV positive person had assaulted an HIV negative person.

Regarding risk, the authors quote seroconversion rates of $0.1 \%-0.2 \%$ after vaginal and $0.1 \%-3 \%$ after anal intercourse. The rate is probably higher in assault because of the greater incidence of traumatic penetration. However, these are not the risks of the adolescent in front of you. The risk for the adolescent is multiplied by the fractional chance that the assailant has HIV. Thus, in sub-Saharan Africa I would want HIV PEP but in rural England I would feel very differently. None of the New York group knew the HIV status of their assailant—and if they did I would question whether this information should be relied upon. The prevalence of HIV in any given population demonstrates extreme regional, gender, and lifestyle variation. The PHLS currently estimates on overall prevalence in the UK of 1:1000. If prevalence rates reflect attendances for care then the prevalence is about 20 times greater in London than in any other region and much greater still in black Africans. ${ }^{3}$

Regarding benefit, the authors provide an honest review. There is no direct evidence. The benefit quoted for occupational HIV PEP (a fivefold risk reduction) is based on a sophisticated case-control study. While Cardo and colleagues made every effort to optimise their data, the sample of cases is small and the controls are retrospective. ${ }^{4}$ Certainly PEP should not be regarded as a proved and effective treatment for occupational exposure, and any extrapolation to the non-occupational setting can only be based on (reasonable) assumptions.

Anyone who has provided health care to this group of patients is well aware of the problems. A typical recommendation is a six week to three month course of triple therapy. Most patients report significant side effects and some are unable to work or attend school for the duration of treatment. There have been two case reports of liver failure requiring transplantation. Compliance is poor.

It is this author's view from the front line that the complexity of individual risk assessment, the lack of evidence for effectiveness, the severity of side effects, and the multiple factors that determine compliance, make each decision about HIV PEP unique. It is no coincidence that guidelines are few and nonprescriptive. If you have immediate access to a genitourinary clinic or advice then discuss early. If not, then don't let concern over the very low risk of HIV blind you to the far more prevalent issues of chlamydial infection, psychological trauma, and child protection. Consider a baseline HIV test or at least saving some serum for future reference.

Emerg Med J 2004;21:403.

doi: $10.1136 / \mathrm{emj} .2003 .005686$

Correspondence to: Dr J Crossley, Sheffield Children's Hospital, Western Bank, Sheffield S10 3TA, UK; i.crossley@sheffield.ac.uk

\section{REFERENCES}

1 Merchant RC, R Keshavarz R, Low C. HIV postexposure prophylaxis provided at an urban paediatric emergency department to female adolescents after sexual assault. Emerg Med J 2004;21:449-51.

2 Giele C, Maw R, Carne CA, et al. Post-exposure prophylaxis for non-occupational exposure to HIV: current practice and opinions in the UK. Sex Transm Infect 2002;78:130-2.

3 Public Health Laboratory Service. HIV and AIDS: epidemiology. London: Public Health Laboratory Service, 2003. (http://www.phls.org.uk).

4 Cardo D, Culver DH, Ciesielski CA, et al. A casecontrol study of HIV seroconversion in health care workers after percutaneous exposure. N Engl J Med, 1997;337:1485-90. 